\title{
Antecedentes poblacionales de Chaptalia exscapa (Pers.) Baker var. chilensis (DC.) Burkart en la provincia de Talca, Chile central
}

\section{Population data of Chaptalia exscapa (Pers.) Baker var. chilensis (DC.) Burkart in Talca Province, central Chile}

\author{
Persy Gómez ${ }^{1}$, Steffen Hahn ${ }^{1}$, José San Martín ${ }^{2 *} \&$ Michail Belov ${ }^{3}$ \\ 'Jardín Botánico, Universidad de Talca, Casilla 747, Talca, Chile. \\ ${ }^{2}$ Instituto de Ciencias Biológicas, Universidad de Talca, Casilla 747, Talca, Chile. \\ ${ }^{3}$ Lo Patricio, Pelarco, Chile. \\ *jsanmart@utalca.cl
}

\begin{abstract}
We examine the distribution and ecology of Chaptalia exscapa (Pers.) Baker var. chilensis (DC.) Burkart in the Talca Province, Maule Region. It is reports new data related to the morphology of the plant and the presence of Chaptalia in four new localities in the Maule Region. The populations present a high vulnerability due low population size and disturbed habitat. Flowering and fructification of individuals is scanty.
\end{abstract}

Chaptalia (Asteraceae) es un género descrito por E.P. Ventenat en el año 1800 presente en América (Heywood et al. 2007, Keller 2013). Incluido en la subfamilia Mutisioideae está representado por hierbas perennes que para el cono sur incluyen a 13 especies con dos categorías infraespecíficas (Zuloaga \& Morrone 1999). De este grupo Chaptalia exscapa comprende las variedades exscapa con distribución en Argentina, Brasil y Uruguay y la var. chilensis registrada solamente para Chile con carácter endémico (Marticorena \& Quezada 1985, Zuloaga \& Morrone 1999, Moreira-Muñoz et al. 2012).

La presencia de Chaptalia exscapa (Pers.) Baker var. chilensis (DC.) Burkart abarca desde Valparaíso a la Araucanía (Moreira-Muñoz et al. 2012) en coincidencia con el área de riqueza de endemismos (Myers et al. 2000). Sin embargo, esta área es reconocida por la histórica presión de uso, transformación del paisaje y sustitución de la vegetación natural principalmente por plantaciones comerciales (Bustamante \& Grez 1995).

Si bien la presencia de Chaptalia exscapa var. chilensis para la Región del Maule es reconocida, las recolecciones son aún reducidas como lo demuestran las únicas dos muestras para la región depositadas en el herbario de la Universidad de Concepción (CONC 167749 y CONC 171460), y la ausencia en el Museo Nacional de Historia Natural (SGO). Esta ausencia de registros, supuestamente, puede tener su origen en la baja abundancia, reducido tamaño de las plantas, carencia de uso y falta de muestreos sistemáticos en la Región del Maule.

Considerando el escenario anteriormente descrito $\mathrm{y}$ la escasa información de la distribución y ecología para la provincia de Talca, Región del Maule, se propone investigar la presencia de Chaptalia exscapa var. chilensis, con descripción del hábitat y fenología reproductiva.

En la primavera de 2013 se trabajó en cuatro nuevas poblaciones de Chaptalia localizadas en la Provincia de Talca: una en Coipue (Pencahue), dos en Tabunco (Curepto), Cordillera de la Costa, y Libueno (Pelarco) de la Precordillera andina (Fig. 1).

El hábitat de Chaptalia exscapa var. chilensis está representado por bosque y matorral esclerófilo siempreverde degradado con especies como Aristotelia chilensis (Molina) Stuntz y Lithrea caustica (Molina) Hook. \& Arn., así como márgenes de remanentes de bosques de Nothofagus glauca (Phil.) Krasser. En 30 individuos como máximo de cada población, elegidos al azar, se fotografiaron y describieron los siguientes caracteres morfológicos: a) disposición y número de hojas, b) número de cabezuelas y c) diámetro de la roseta radical. La fenología reproductiva se focalizó en la presencia de flores y/o frutos. Finalmente en 3 cuadrantes de $1 \mathrm{~m}^{2}$ de cada población, se determinó la densidad promedio y distribución espacial según Morishita (1962).

La vegetación nativa de los sitios se presenta degradada en cobertura e integridad con claros favorables para el pastoreo de fauna doméstica. Tres poblaciones de Chaptalia exscapa var. chilensis se encuentran en matorrales esclerófilos con exposición de sol directo por la mañana y algo de sombra por la tarde (Fig.2A). Contrariamente una de las poblaciones de Tabunco con individuos dispersos y baja densidad se encuentra bajo sombra parcial y plantación joven de Pinus radiata $\mathrm{D}$. Don.

Los ejemplares de Chaptalia exscapa var. chilensis presentan hojas arrosetadas, discolóreas y consistencia membranosa (Reiche 1905, Burkart 1944) (Fig. 2B). Desde el punto de vista de la morfología se observaron diferencias entre los individuos tanto en la densidad foliar por roseta como en el número de cabezuelas y diámetro de la roseta 


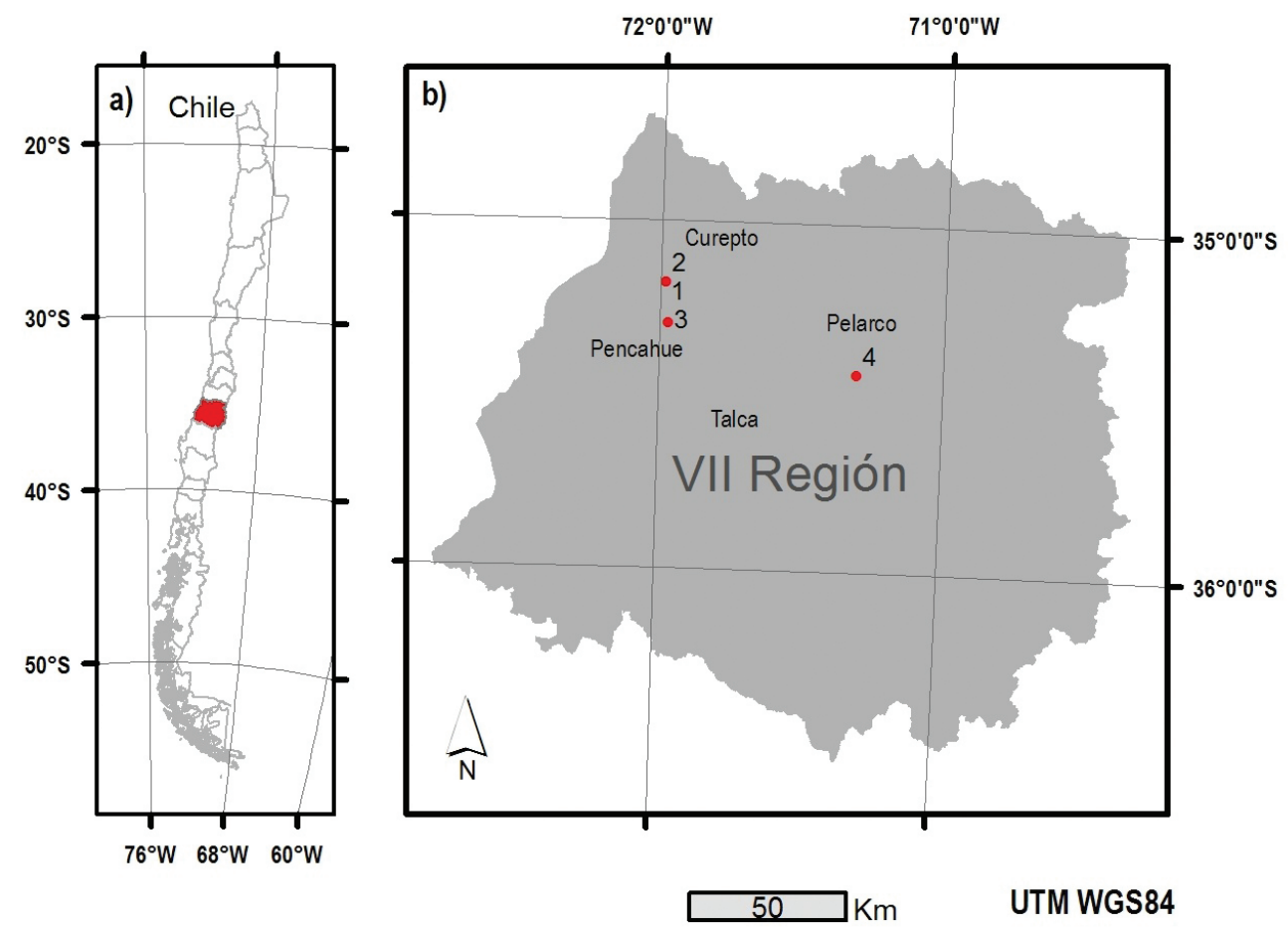

Figura 1. Sitios de estudio de Chaptalia exscapa (Pers.) Baker var. chilensis (DC.) Burkart en la Región del Maule. a) Mapa de Chile caracterizando la Región del Maule. b) Aproximación de la Región del Maule con 4 localidades en que se ha encontrado Chaptalia: 1) Tabunco en plantación, Curepto. 2) Tabunco en matorral, Curepto. 3) Cruce a Coipue -matorral, Pencahue y 4) Libueno en matorral, Pelarco. Figure 1. Sites of study of Chaptalia exscapa (Pers.) Baker var. chilensis (DC.) Burkart in the Maule Region. a) Map of Chile characterizing the Maule Region. b) Close-up of the Maule Region with four locations of Chaptalia: 1) Tabunco plantation, Curepto. 2) Tabunco shrub, Curepto. 3) Crossing to Coipue shrub, Pencahue y 4) Libueno shrub, Pelarco.

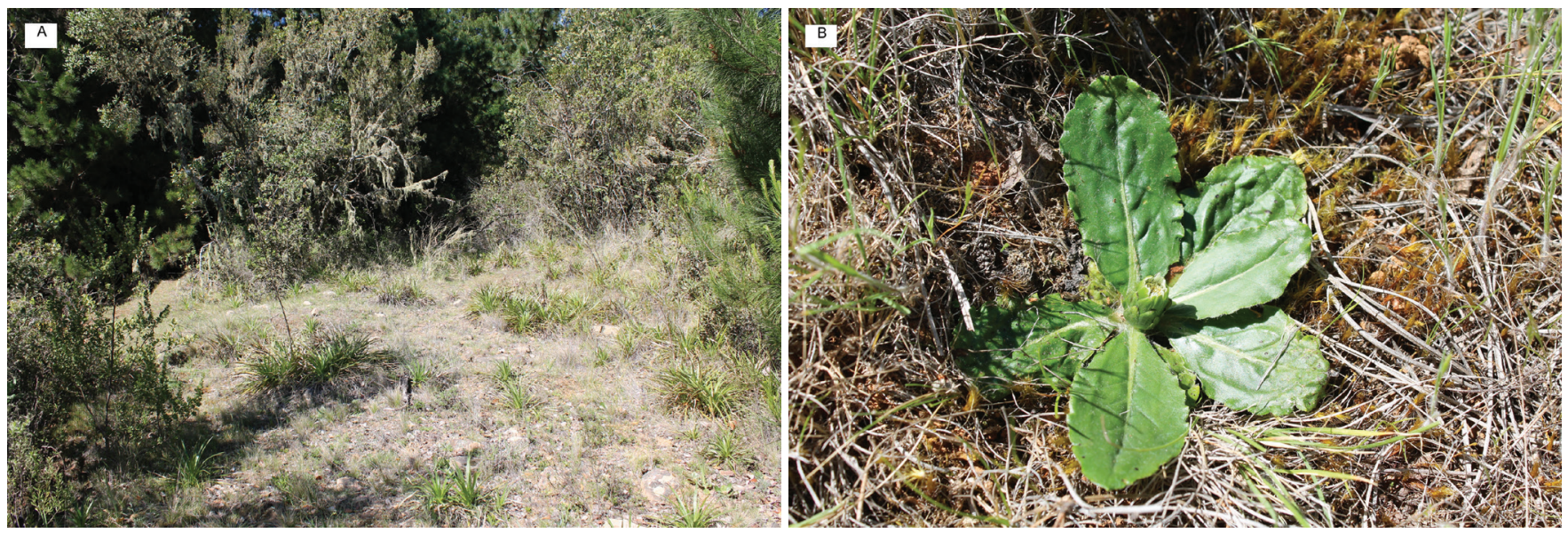

Figura 2. A) Hábitat típico y B) vista de Chaptalia exscapa (Pers.) Baker var. chilensis (DC.) Burkart, matorral esclerófilo rodeado por plantaciones forestales.

Figure 2. A) Typical habitat and B) view of Chaptalia exscapa (Pers.) Baker var. chilensis (DC.) Burkart, sclerophyll shrub surrounded by forest plantations. 
TABLA I. Localidades, tipo de hábitat y antecedentes morfológicos de individuos de Chaptalia exscapa (Pers.) Baker var. chilensis (DC.) Burkart en la Región del Maule.

TABLE I. Localities, type of habitat and morphologic precedents of individuals of Chaptalia exscapa (Pers.) Baker var. chilensis (DC.) Burkart in the Maule Region.

\begin{tabular}{|c|c|c|c|c|c|c|}
\hline LOCALIDADES & $\begin{array}{l}\text { COORDENADAS } \\
\text { GEOGRÁFICAS }\end{array}$ & $\begin{array}{c}\text { TAMAÑO POBLACIONAL } \\
\text { (InDIVIDUOS) }\end{array}$ & $\begin{array}{l}\text { NÚMERO } \\
\text { PLANTAS }\end{array}$ & $\begin{array}{c}\text { NÚMERO } \\
\text { HOJAS }\end{array}$ & $\begin{array}{l}\text { NÚMERO } \\
\text { CABEZUELAS }\end{array}$ & $\begin{array}{l}\text { DiÁMETRO ROSETA } \\
\text { RADICAL (CM) }\end{array}$ \\
\hline Libueno-Pelarco (matorral) & $35^{\circ} 25^{\prime} 59^{\prime \prime} \mathrm{S} ; 71^{\circ} 17^{\prime} 5^{\prime \prime O}$ & 200 & 30 & 4 a 13 & 0 a 2 & 7.5 a 14.5 \\
\hline Tabunco-Curepto (plantación) & $35^{\circ} 10^{\prime} 38^{\prime \prime} \mathrm{S} ; 71^{\circ} 58^{\prime} 48^{\prime \prime} \mathrm{O}$ & 4 & 4 & 8 a 10 & 0 a 1 & 10.5 a 19 \\
\hline Tabunco-Curepto (matorral) & $35^{\circ} 10^{\prime} 38^{\prime \prime} \mathrm{S} ; 71^{\circ} 58^{\prime} 53^{\prime \prime} \mathrm{O}$ & 20 & 20 & 4 a 9 & 0 a 1 & 9 a 22 \\
\hline Cruce a Coipue (matorral) & $35^{\circ} 17^{\prime} 38^{\prime \prime} \mathrm{S} ; 71^{\circ} 58^{\prime} 10^{\prime \prime} \mathrm{O}$ & 40 & 30 & 5 a 11 & 0 a 2 & 7 a 14.5 \\
\hline
\end{tabular}

radical (Tabla I). El número de hojas varía entre 4-13, mientras que el diámetro de la roseta fluctúa entre 7-22 $\mathrm{cm}$. Finalmente, el número de cabezuelas varía de uno a dos, antecedente no reportado por Burkart (1944) y Reiche (1905).

En la fenología y fase reproductiva se observó que los primeros botones florales de Chaptalia se desarrollan a fines de septiembre con antesis a comienzos de octubre. La formación, maduración y dispersión de frutos se presenta principalmente en octubre. Aunque la floración y fructificación son procesos temporalmente cortos, entre los individuos de cada población y entre los diferentes sitios no presentan sincronía. Para Libueno el $20 \%$ de los individuos formaron flores y frutos, un $32 \%$ en Coipue, un $10 \%$ en el matorral esclerófilo de Tabunco y solamente 2 de 4 individuos en la población bajo la plantación de pino. No se observaron polinizadores en los capítulos florales de Chaptalia exscapa var. chilensis. Si bien este resultado es un avance en el conocimiento de la especie, no está de acuerdo con lo propuesto por Torres \& Galetto (2008) en cuanto a que las especies de Asteráceas con floración de pocas semanas, simultáneamente ofrecen una gran cantidad de inflorescencias por planta como de flores por capítulo, por lo cual presentarían los ensambles de visitantes más diversos y las mayores frecuencias de visitas.

Los tamaños poblacionales de Chaptalia exscapa var. chilensis son bajos y desiguales, encontrándose en Libueno 200 individuos, 24 en Tabunco y 40 en Coipue. La densidad promedio por $\mathrm{m}^{2}$ varía desde 1 y 6 para las poblaciones de Tabunco, 12 Libueno y 2 individuos para Coipue. La distribución horizontal de las poblaciones es fragmentada con subpoblaciones con disposición agregada.

La condición de hábitat de las poblaciones entre los sitios es heterogénea aunque coinciden en el patrón de distribución y reducido tamaño de las poblaciones. Una situación crítica se observa en Tabunco con una población de sólo cuatro individuos bajo pinos y que podría derivar en una confirmación a lo planteado por Gilpin \& Soulé (1986), en cuanto a que especies con reducidos tamaños poblacionales en ambientes hostiles presentan mayor probabilidad de extinción.

Utilizando los Criterios de UICN versión 3.1 (2001), estimamos que este taxón puede ser categorizado como En Peligro para la Provincia de Talca dado que:

EN B1ab(iii); C2a(i); D

B1: Extensión de presencia estimada menor a $5.000 \mathrm{~km}^{2}$. Estimada en $415 \mathrm{~km}^{2}$.

B1a: se conoce en menos de 5 localidades

B1b(iii): se estima que hay disminución del área, extensión y/o calidad del hábitat principalmente por el cambio de uso del suelo hacia plantaciones de carácter productivo.

C2a(i): tamaño de la población estimada en menos de 2500 individuos maduros y su disminución proyectada indicando que ninguna subpoblación contiene más de 250 ejemplares maduros. D: Se estima que el tamaño de la población es menor de 250 individuos maduros.

Sin embargo, son necesarios una mayor cantidad de muestreos para ratificar este resultado. Para la Provincia de Talca, se concluye que las poblaciones de Chaptalia, al ocupar ambientes alterados en un paisaje antropogenizado con bajos tamaños poblacionales, se encuentra en una condición desfavorable para su sobrevivencia y expansión del área in situ.

\section{AGRADECIMIENTOS}

Se agradece el apoyo de la DI, Jardín Botánico e Instituto de Ciencias Biológicas de la Universidad de Talca, así como a Alicia Marticorena y Gloria Rojas, herbarios de CONC y $\mathrm{SGO}$, respectivamente. También por el material bibliográfico a Gastón Sotes, Universidad de Concepción. A Yony Ormazábal del Centro de Geomática de la Universidad de Talca. A los revisores anónimos que con sus comentarios han contribuido a mejorar el manuscrito. 


\section{BIBLIOGRAFÍA}

Burkart, A. 1944. Estudio del género de Compuestas Chaptalia con especial referencia a las especies argentinas. Darwiniana 6(4): 505-594.

Bustamante, R. \& A. Grez. 1995. Consecuencias ecológicas de la fragmentación de los bosques nativos. Ambiente y Desarrollo 11: 58-63.

Gilpin, M.E. \& M.E. SoulÉ. 1986. Minimum viable populations: processes of species extinction. In: M.E. Soulé (ed.), Conservation Biology: The Science of Scarcity and Diversity. Sinauer, Sunderland, MA, pp. 19-34.

Heywood, R., K. Brummitt, A. Culham \& O. Seberg. 2007. Flowering plant families of the world. Firefly Books Ltd. $424 \mathrm{pp}$.

Keller, H.A. 2013. No soy feliz: origen, usos y agencia social de Chaptalia nutans (Asteraceae) según los guaraníes de Misiones, Argentina. Bonpladia 22(2): 171-180.

Marticorena, C. \& M. Quezada. 1985 Catálogo de la flora vascular de Chile. Gayana Botánica 42(1-2): 1- 152.
Moreira-Muñoz, A., V. Morales \& M. Muñoz-Schick. 2012. Actualización sistemática y distribución geográfica de Mutisioideae (Asteraceae) de Chile. Gayana Botánica 69(1): 9-29.

MorishitA, M. 1962. Id-index: A measure of dispersion of individuals. Researches Population Ecology. 4: 1-7.

Myers, N., R.A. Mittermeier, C.G. Mittermeier, G.A.B. DA Fonseca \& J. Kent. 2000. Biodiversity hotspots for conservation priorities. Nature 403: 853-858.

Reiche, K. 1905. Estudios críticos sobre la Flora de Chile. Anales de la Universidad de Chile, vol. 4, pág. 364-365.

Torres, C. \& L. Galetto. 2008. Importancia de los polinizadores en la reproducción de Asteraceae de Argentina central. Acta Botánica Venezuelica 31(2): 473-494.

UICN. 2001. Categorías y criterios de la lista roja de la UICN: versión 3.1. Comisión de supervivencia de especies de la UICN. UICN, Gland, Suiza \& Cambridge, Reino Unido. ii + 33pp.

Zuloaga, F. \& O. Morrone. 1999. Catálogo de las Plantas Vasculares de la República Argentina (Dicotyledoneae). Monographs in Systematic Botany from the Missouri Botanical Garden 74: 98-353.

Recibido: 13.12.14

Aceptado: 16.01 .15 